\title{
Fast Cooling Techniques for Gravitational Wave Antennas
}

\author{
S.R. Furtado and O. D. Aguiar \\ Instituto Nacional de Pesquisas Espaciais - INPE \\ Avenida dos Astronautas, 1758 \\ São José dos Campos, SP 12227-010, Brazil \\ e-mail: sergio@das.inpe.br, odylio@das.inpe.br
}

\begin{abstract}
The resonant-mass technique for the detection of Gravitational Waves may involve, in the near future, the cooling of very large masses (about 100 tons) from room temperature $(300 \mathrm{~K})$ to extreme cryogenic temperatures $(20 \mathrm{mK})$. To cool these detectors down to cryogenic temperatures an exchange gas (helium) is used, and the heat is removed from the antenna to the cold reservoir by thermal conduction and natural convection. With the current technique, cooling times of about one month can be obtained for cylindrical bar antennas of 2.5 tons. Should this same technique be used to cool a 100-ton spherical antenna the cooling time would be about 10 months, making the operation of these antennas impracticable. In this work we study the above-mentioned cooling technique and others, like thermal switching and forced convection from room temperature down to liquid nitrogen temperature $(77 \mathrm{~K})$ using an aluminum, $19 \mathrm{~kg}-25 \mathrm{~cm}$-diameter, truncated icosahedron.
\end{abstract}

\section{$1 \quad$ Introduction}

Cooling the Nautilus cylindrical bar antenna (Al 5056, $3 \mathrm{~m}$ long, $0.6 \mathrm{~m}$ diameter, $2300 \mathrm{~kg}$ ), from room temperature $(300 \mathrm{~K})$ to the liquid nitrogen temperature $(77 \mathrm{~K})$, takes about 3 weeks using 8000 liters of liquid nitrogen and about one week to achieve $4.2 \mathrm{~K}$, using about 5000 liters of helium ${ }^{1}$. The cooling time of Nautilus shows that the main thermal resistance is due to the thermal conductivity of the gas, and an extrapolation to a $3 \mathrm{~m} \mathrm{CuAl}$ sphere (107 tons) yields several months for the cooling time ${ }^{2}$. This cooling time can be significantly reduced if the heat is removed from the antenna through forced convection ${ }^{3}$ as demonstrated by the Dutch Group for a $15 \mathrm{~cm} \mathrm{CuAl}(6 \%)$ sphere ${ }^{4}$. Another technique, named thermal switching, can also be used for fast cooling these antennas, as we will show below.

\subsection{Heat exchange by forced convection}

Forced convection consists of removing the heat from the antenna, at an initial temperature $T_{A}$, through direct contact with a cold gaseous fluid, at temperature $T_{g a s}<T_{A}$ and pressure $P_{\text {gas }}$, whose movement is artificially induced so that its flow will be forced around the surface of the sphere. Since the outflow velocity of the fluid in free convection is much lower than in forced convection, the heat transfer by forced convection will be much higher than the one by free convection. Using this technique, the Leiden Group cooled a $65 \mathrm{~cm}-$ 
1.15ton- $\mathrm{CuAl}(6 \%)$ antenna down to $1.8 \mathrm{~K}$ within 3.5 days $^{5}$, using forced convection of helium gas. The heat exchange by convection can be expressed by:

$$
q=h \cdot S \cdot\left(T_{A}-T_{\text {gas }}\right)
$$

Where $\boldsymbol{q}$ is the rate in which the heat is removed from sphere, $\boldsymbol{h}$ is the heat transfer coefficient and $S$ is the superficial area of the antenna. Using the lumped thermal capacitance method ${ }^{6}$, we can obtain the following expression for the transient temperature of the antenna:

$$
T=T_{\text {gas }}+\left(T_{A}-T_{\text {gas }}\right) \exp \left[-\left(\frac{h S}{\rho V c}\right)\right]
$$

Where $T$ represents the antenna temperature at time $t, h$ is the average heat transfer coefficient between the antenna and the gas in movement, $\rho$ and $c$ are the density and specific heat of sphere respectively, and $V$ is the volume of sphere.

One of the biggest problems in studying heat exchange by convection is the definition of $\boldsymbol{h}$, which should be obtained either analytically or experimentally. Convection depends on viscosity, thermal conductivity, specific heat, fluid density and velocity of the fluid. We may evaluate the heat transfer coefficient $h$ using the following expression for the average dimensionless Nusselt number $\left(N u_{D}\right)$ for a gas flowing around a sphere ${ }^{7}$ :

$$
N u_{D}=\frac{h D}{k}=2+\left(0,4 \operatorname{Re}_{D}^{0,5}+0,06 \operatorname{Re}_{D}^{2 / 3}\right) \operatorname{Pr}^{0,4}\left(\mu_{\text {gas }} / \mu_{A}\right)^{1 / 4}
$$

Where $D$ represents the diameter of the sphere, $k$ is the thermal conductivity of the gas, $P r$ and $R e_{D}$ are the dimensionless Prandtl and Reynolds parameters respectively and, $\mu_{\text {gas }}$ and $\mu_{A}$ are the viscosity of gas evaluated at $T_{\text {gas }}$ and $T_{A}$ (initial surface temperature of the sphere) respectively. In a general way, the Nusselt number is related to the thermal aspects of the cooling processes and the Reynolds number to the hydrodynamic ones.

\subsection{Heat exchange by thermal switching}

The thermal switching ${ }^{8}$ technique consists of removing the heat from the antenna through thermal conduction (thermal contact) between the antenna and a cold body, which in turn is kept in contact with a cold bath.

The problem of cooling a resonant mass antenna by thermal switching can be represented as following: a cylindrical conductive bar connects a cold source, at constant temperature $\boldsymbol{T}_{\boldsymbol{B}}$, at one end, to the antenna, which is initially at the temperature $\boldsymbol{T}_{\boldsymbol{A}} \gg \boldsymbol{T}_{\boldsymbol{B}}$ at the other end. Therefore, heat flows from the antenna to the cold source through the metallic bar. The rate $\boldsymbol{q}$, in which the heat is removed from sphere, can be expressed by: 


$$
q=-\frac{k_{B} \cdot S_{B} \cdot\left(T-T_{B}\right)}{L_{B}}
$$

Where $\boldsymbol{k}_{\boldsymbol{B}}$ is the heat transfer coefficient by thermal conduction, $\boldsymbol{S}_{\boldsymbol{B}}$ and $L_{B}$ are the transversal area and length of the conduction bar respectively, and $T$ represents the antenna temperature at time $\mathrm{t}$. So the expression for the transient temperature of the antenna can be written by:

$$
T=T_{B}+\left(T_{A}-T_{B}\right) \exp \left[-\left(\frac{k_{B} S_{B}}{L_{B} \rho V c}\right)\right]
$$

Where $\rho, V$ and $c$ are the density, volume and specific heat of sphere respectively.

As the thermal conductivity for metals is much higher than for gases, heat transfer by metal conductivity becomes much more efficient than by gas conductivity. So, we expect with the technique described above to obtain competitive cooling time if compared with the time obtained by the forced convection technique.

\section{Experimental Setup}

All tests and set-ups of this experiment were conducted in the facilities of the Laboratory of Integration and Testing (LIT) of the Brazilian National Institute for Space Research (INPE).

The antenna (a truncated icosahedron (buckyball) made of aluminum with a diameter of $25 \mathrm{~cm}$ and $19 \mathrm{~kg}$ of weight) was cooled down from room temperature to the boiling point of liquid nitrogen (77.4K). Nitrogen gas was used as the heat exchange fluid. Figure 1 shows the antenna used to study of fast cooling techniques.

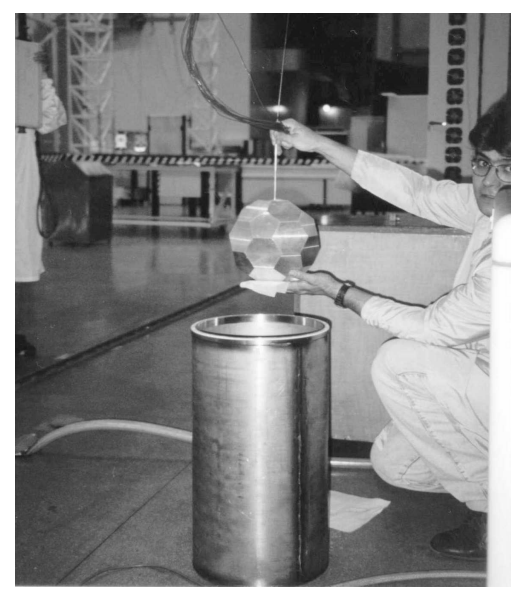

Figure 1: Detail of the experimental chamber and antenna used for the tests. This antenna is a truncated icosahedron (buckyball) made of aluminum with a diameter of $25 \mathrm{~cm}$ and $19 \mathrm{~kg}$ of weight 
A 1.0-mm-diameter stainless steel cable suspended the buckyball inside a stainless steel vacuum chamber, which has an internal diameter of $31.5 \mathrm{~cm}$. This vacuum chamber was immersed in a liquid nitrogen bath, which maintained the temperature of the chamber walls at approximately $78.5 \mathrm{~K}$ (close to the boiling temperature of liquid nitrogen). There were two pipes inside the chamber, one for the inflow and the other for the outflow nitrogen gas (78K). Eight type T thermocouples were employed to monitor the buckyball temperature, and another 23 thermocouples were used for the cryogenic system. These sensors have an accuracy of $\pm 0.7^{\circ} \mathrm{C}$ for temperatures above and $\pm 1.2^{\circ} \mathrm{C}$ for temperatures below $0^{0} \mathrm{C}$.

The following heat exchange configurations were analyzed:

- Heat exchange due to thermal radiation, gas conductivity and natural gas convection: during this cooling phase the antenna was kept suspended (far from the conduction bar). The internal pressure of the vacuum chamber was maintained at approximately 2 mbar;

- Heat exchange due to thermal radiation, metal conductivity, gas conductivity and natural gas convection: during this cooling phase the antenna was maintained in touch with a metallic bar coupled to the vacuum chamber, ensuring the constant temperature of the metallic bar at $78.5 \mathrm{~K}$. The internal pressure of the vacuum chamber was maintained at approximately 2 mbar;

- Heat exchange due to thermal radiation, gas conductivity, natural and forced convection: during this cooling phase the antenna remained suspended (far from the metallic bar). Cold nitrogen gas was injected by the lower tube, flowing around the buckyball to the upper part of the vacuum chamber (and being collected by the upper tube), causing heat exchange between the antenna and the cold gas. The gas flow was kept constant at $20 \mathrm{Nl} / \mathrm{min}$. This corresponds to a gas flow velocity of $2.4 \%$ of the speed of sound in nitrogen gas at $78 \mathrm{~K}\left(\mathrm{~V}_{\text {sound }(78 \mathrm{~K})} \sim 180.0 \mathrm{~m} / \mathrm{s}\right)^{9}$ inside the pipes, which have $10 \mathrm{~mm}$ of internal diameter. The chamber's internal pressure was maintained at approximately 420 mbar (below normal atmospheric pressure - 1000 mbar), avoiding the condensation of the cold nitrogen gas inside the chamber.

The figures 2 and 3 shows a schematic drawing of the setup inside the cryostat to study fast cooling techniques and a few details of the setup inside the vacuum chamber, respectively.

\section{Results}

Figure 4 shows a comparison between the three studied cases in the 300-80K range of temperature.

\section{Conclusion}

Comparing the cooling times of the buckyball in Figure 4 for all three cases, it is apparent that the forced convection and thermal switching techniques were significantly more 
efficient than the natural convection technique. The cooling times were, respectively, 30h, $70 \mathrm{~h}$, and 200h.

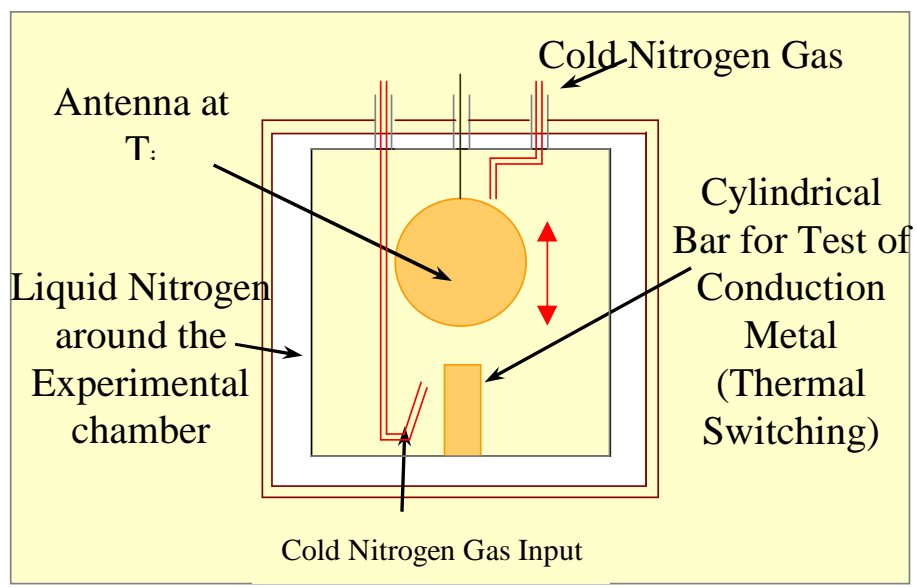

Figure 2: Experimental assembly for Heat Exchange by Thermal Radiation, Gas/Metal Conduction, and Natural/Forced Convection. The vacuum chamber was submerged in a liquid nitrogen bath, keeping the wall temperature at $78.5 \mathrm{~K}$ and in low vacuum $(\sim 420$ mbar). During the tests it was possible to raise or lower the antenna in order to have it in thermal coupling, or not, with the metallic bar placed on the bottom of the tank.

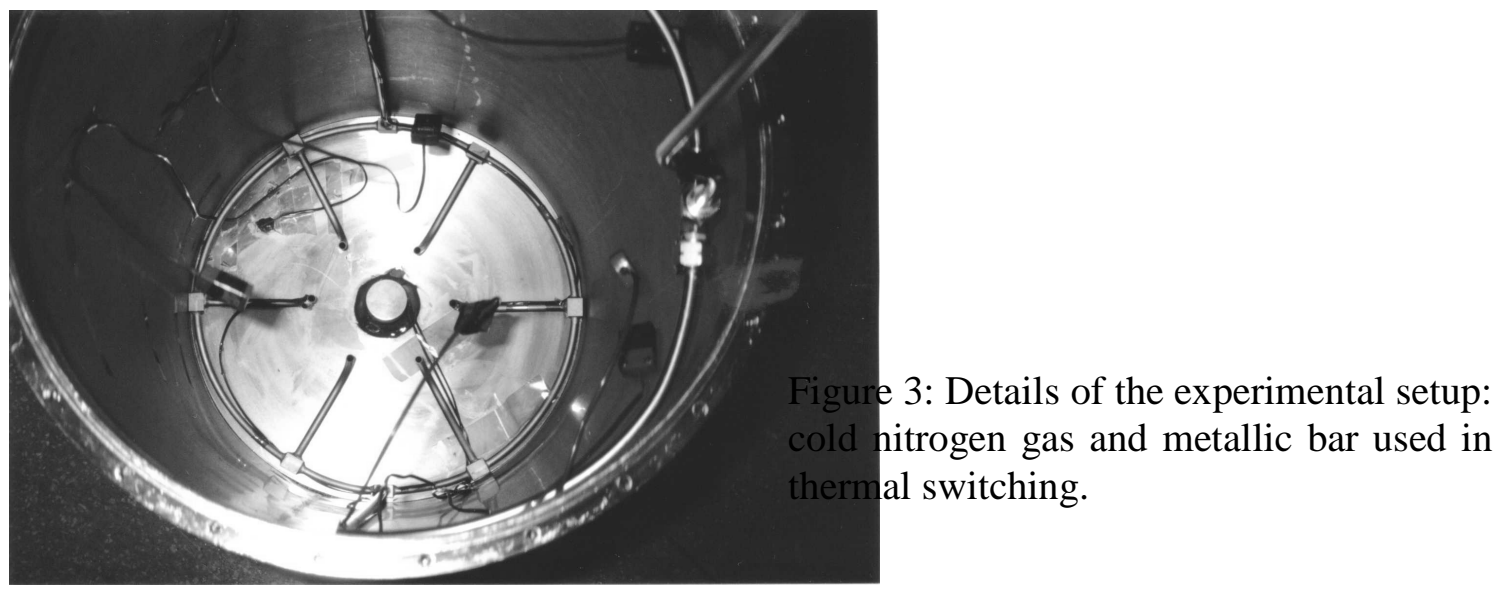

The poor performance obtained with the present experiments to cool a $25-\mathrm{cm}$ diameter Al sample by forced convection is due to the bad thermal conductivity of the Nitrogen gas at temperatures close to $80 \mathrm{~K}$ (the gas temperature), even if the pressure is kept below 1 bar. This performance can be improved significantly if we replace the gas used for heat exchange from nitrogen to helium. The conductivity of helium gas at $80 \mathrm{~K}$ is about 7.5 times larger then the one for nitrogen gas at this same temperature. This and other factors cause the coefficient of heat exchange by forced convection of helium gas to be about 6.5 times larger then the one for nitrogen gas causing the cooling time from $300 \mathrm{~K}$ to $80 \mathrm{~K}$, by forced convection, to be about 4.5 hours. Evidently this is function of the flow used. 
We believe it is possible to improve the cooling efficiency in the case of thermal switching if we increase the contact area between the cylindrical bar and the antenna, through a better superficial finish. We calculated that in the case analyzed in Figure 4 the effective area of contact between the buckyball and the metal rod was only about $10 \%$ of the rod cross section. Another possibility to increase the efficiency of the thermal switch would be to replace the $\mathrm{CuAl}(6 \%)$ bar for an OFHC copper bar, which has a coefficient of thermal conduction 5 times larger in the 300-80K temperature range. A further increase on the effective area of the bar to $30 \%$ of the geometric area would cause a total decrease of 15 times the cooling time (from $70 \mathrm{~h}$ to about $4.5 \mathrm{~h}$ ), making it competitive with the forced convection technique.

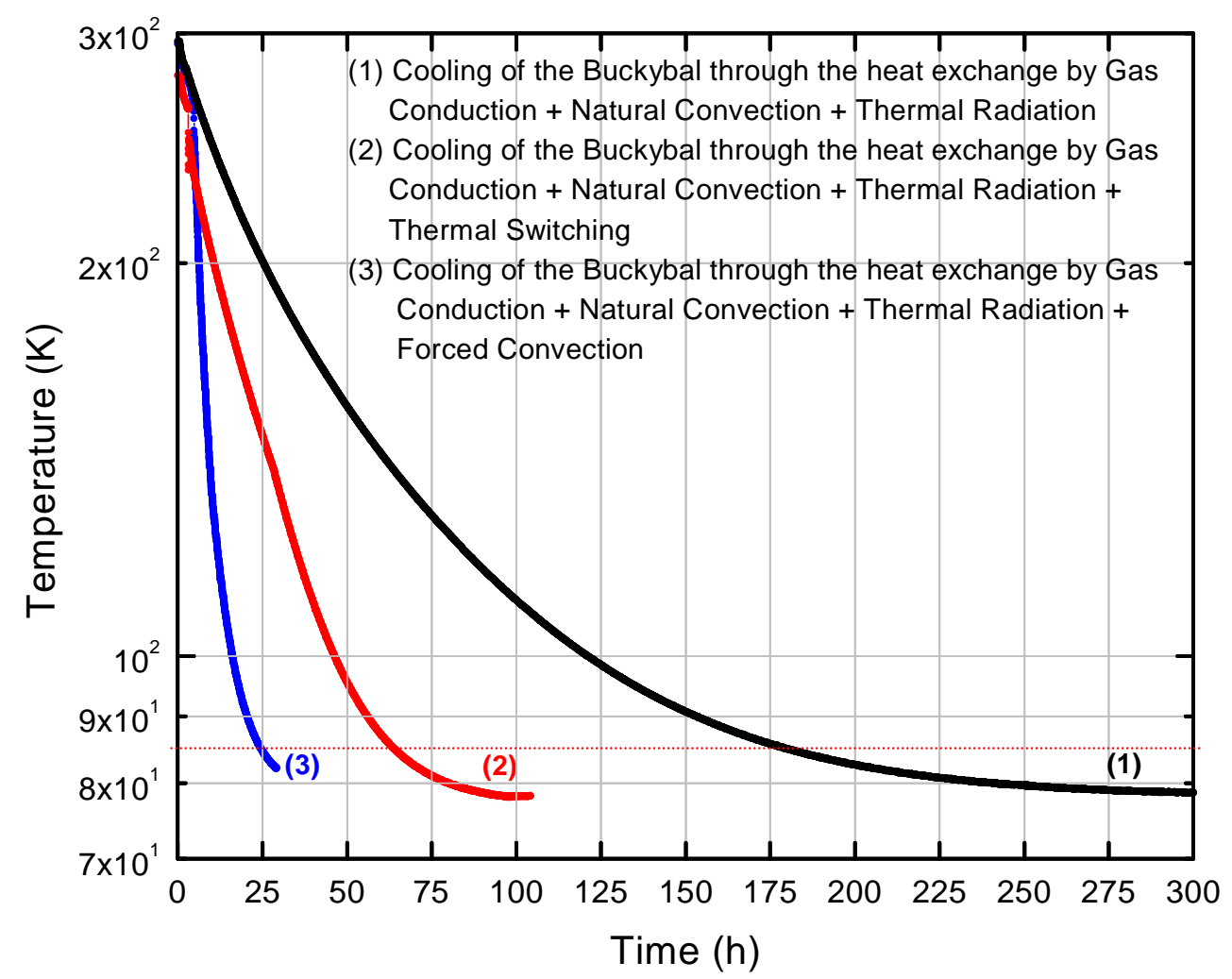

Figure 4 - Comparison between the three studied cases (gas conduction, thermal switching and forced convection). The $300-80 \mathrm{~K}$ range of temperature was used. 


\section{Acknowledgments}

This work has been supported by FAPESP under grants No. 1998/13468-9 and \# 2001/04189-3, and by CNPq under grant No. 300619/92-8. We thank Prof. Nei Oliveira for useful discussions.

We would like to thank Dr. Clóvis Solano Pereira, Dr. Ézio Castejon Garcia and Dr. Marcio Bueno and the technical staff of the Laboratory of Integration and Testing (LIT). We also would like to thank one of the referees for very useful comments and suggestions.

\section{References}

\footnotetext{
${ }^{1}$ Astone P et al 1991 First cooling below $0.1 \mathrm{~K}$ of the new gravitational-wave antenna "NAUTILUS" of the Rome group, Europhys. Lett. 16 (3) 231-5

${ }^{2}$ Frossati G et al 1994 Cryogenic aspects of cooling large masses to millikelvin temperatures: aplication to a 100 ton mK spherical gravitational wave detector ICEC 15 Proceeding (Genova, Italy)

${ }^{3}$ Frossati G 1997 A 100 TON 10 mK Spherical gravitational wave detector, Proc. first international workshop on Omnidirectional gravitational radiation observatory ed W F Velloso Jr., O D Aguiar and N S Magalhães (Singapore: World Scientific) pp 163-8

${ }^{4}$ De Waard A., Gottardi L and Frossati G 2000 Cooling and Quality Factor of a Small CuAl6\% Sphere Proc. of the Ninth Marcel Grossmann Meeting (Rome) to be published

${ }^{5}$ De Waard A, Gottardi L and Frossati G Progress Report 2001: First Cooldown, in this Proceedings

${ }^{6}$ Özisik M N 1985 Heat Transfer: a basic approach, MCGraw-Hill, New York

${ }^{7}$ Whitaker, S.: "Forced Convection Heat Calculations for Flow in Pipes, past Flat Plates, Single Cylinders, and for Flow in Packed Beds and Tubes Bundles", AIChE J., 18: 361 - 371 (1972)

${ }^{8}$ Furtado S R and Aguiar O D 1999 Boletim da Sociedade Astronômica Brasileira (São Paulo), vol. 19(1), p.142

${ }^{9}$ Vennard J K and Street R L 1975 Elementary Fluid Mechanics ed John Wiley \& Sons (New York)
} 